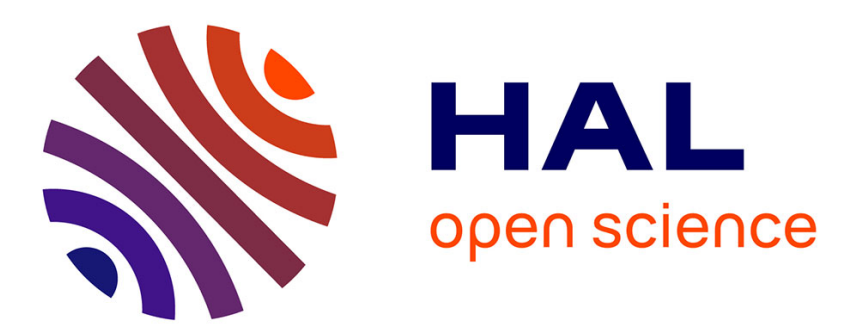

\title{
Tip streaming of a lipid-stabilized double emulsion generated in a microfluidic channel
}

Kristian Torbensen, Charles N Baroud, Sandra Ristori, Ali Abou-Hassan

\section{To cite this version:}

Kristian Torbensen, Charles N Baroud, Sandra Ristori, Ali Abou-Hassan. Tip streaming of a lipid-stabilized double emulsion generated in a microfluidic channel. Langmuir, 2021, 10.1021/acs.langmuir.1c00827 . hal-03260169

\section{HAL Id: hal-03260169 \\ https://hal.sorbonne-universite.fr/hal-03260169}

Submitted on 14 Jun 2021

HAL is a multi-disciplinary open access archive for the deposit and dissemination of scientific research documents, whether they are published or not. The documents may come from teaching and research institutions in France or abroad, or from public or private research centers.
L'archive ouverte pluridisciplinaire HAL, est destinée au dépôt et à la diffusion de documents scientifiques de niveau recherche, publiés ou non, émanant des établissements d'enseignement et de recherche français ou étrangers, des laboratoires publics ou privés. 


\title{
Tip streaming of a lipid-stabilized double emulsion generated in a microfluidic channel
}

Kristian Torbensen,,$^{a, \dagger}$ Charles N. Baroud, ${ }^{b, c}$ Sandra Ristorid ${ }^{d}$ and Ali Abou-Hassan ${ }^{a *}$

a Sorbonne Université, CNRS UMR 8234, Laboratoire PHysico-chimie des Électrolytes et Nanosystèmes InterfaciauX, PHENIX, F-75005 Paris, France.

${ }^{\mathrm{b}}$ LadHyX \& Department of Mechanics, Ecole Polytechnique, CNRS, 91128 Palaiseau, France

${ }^{c}$ Physical Microfluidics \& Bioengineering, Institut Pasteur, 25 Rue du Dr. Roux, 75015, Paris, France

d Chemistry Department \& CSGI, University of Florence, via Della Lastruccia 3,50019, Sesto Fioretino, Italy

\begin{abstract}
Water/oil/water (w/o/w) double emulsions (DEs) are multicompartment structures which can be used in many technological applications and in fundamental studies as models of cells like microreactors or templates for other materials. Herein we study the flow dynamics of water/oil/water double emulsions generated in a microfluidic device and stabilized with the phospholipid 1,2-dimyristoyl-sn-glycero-3-phosphocholine (DMPC). We show that by varying the concentration of lipids in the oil phase (chloroform), or by modulating the viscosity of the aqueous continuous phase, the double emulsions under flow exhibit a rich dynamic behavior. An initial deformation of the double emulsions is followed by tube extraction at the rear end, relative to the flow direction, resulting in pinch off at the tube extremity, by which small aqueous compartments are released. These compartments are phospholipid vesicles as deduced from fluorescence
\end{abstract}


experiments. The overall process can thus be of help to shed light on the mechanical aspects of phenomena such as the budding and fusion in cell membranes.

\section{INTRODUCTION}

Over the past decades, the field of droplet microfluidics has witnessed a rapid evolution. ${ }^{1-2}$ This is in particular due to achievements in the soft lithographic technology, ${ }^{3}$ and in engineering of simple and versatile microfluidic devices built using coaxially aligned capillary tubes. ${ }^{4-5}$ With high control over flow rates and both channel geometry and dimensions, monodisperse multi-compartment droplets, with single or multiple cores can be generated with high throughput for applications in biology, chemistry and nanotechnology. ${ }^{6-9}$ Water/oil/water double emulsions (DEs) are droplets that have attracted widespread interest over about thirty years for applications ranging from the formulation of pharmaceutics to more fundamental studies. ${ }^{4,10-12}$ They can be generated with a narrow size distribution using the two-step emulsification method in microfluidics. ${ }^{10}$ The DEs are used as templates for the preparation of other materials including among others giant phospholipid vesicles, ${ }^{13-15}$ polymersomes, ${ }^{16-17}$ hole-shell particles $^{18}$ or as a model of microreactors for the synthesis of biomaterials ${ }^{13}$ and the study of complex biological processes. ${ }^{19-22}$

Several studies on deformation, breakup and/or tip-streaming of single emulsions in flow

systems have been reported, ${ }^{8,23-25}$ but less on DEs. While the behavior of single emulsions can be described by the interfacial tension and the viscosity ratio between the droplet and the continuous flowing outer fluid, the flow dynamics of multiple emulsions require several additional parameters to account for multiple interfaces and the presence of domain with different viscosities. ${ }^{8}$ We must therefore keep track of the viscosities $(\mu)$ of the three phases: the inner phase $\left(\mu_{\text {in }}\right)$, the middle oil 
phase $\left(\mu_{\mathrm{oil}}\right)$, and the external continuous phase $\left(\mu_{\mathrm{ext}}\right)$, as well as the value of the interfacial tension $(\gamma)$, which we will consider to be the same on both interfaces.

When subject to stress, such as under shear flow conditions in microfluidic channels, deformation of both the core droplet and the shell might occur, either independently or simultaneously. The dynamics of double emulsions under shear flow has been investigated numerically by Hua et $a l .{ }^{26}$ and both numerically and experimentally by Chen et $a l .{ }^{27-28}$ in a Couette geometry. The deformation of the outer droplet (i.e., the middle phase) was shown to be governed by competition between viscous shear stress and interfacial tensions, together with the ratio between the core and outer droplet radii. The deformation of the core was demonstrated to be mainly induced by a vorticity flow within the outer droplet, generated by the shear stress between the middle phase and the external phase. It was furthermore shown, ${ }^{27}$ that at critical capillary numbers, a transformation from a steady shape to a transient deformation eventually resulted in the breakup of the outer droplet. Ma et al. ${ }^{29}$ investigated the core deformation of w/o/w DEs undersheath flow focusing. They emphasized the significance of core and shell viscosities on core deformation, whereas the type of surfactant present at the interfaces apparently was of minor importance. Chunfeng et al..$^{30}$ and Tao et al. ${ }^{31}$ studied numerically the flow dynamics of compound drops in contracting tubes. Similarly, Chen et al. ${ }^{32}$ investigated the deformation and breakup mechanism of double emulsions in a tapered nozzle.

In this manuscript we show that DMPC stabilized DEs elaborated with chloroform as the oil shell exhibits a rich dynamic behavior under flow in a microfluidic channel. Depending on the concentration of DMPC in the oil shell and the concentration of poly-vinyl-alcohol (PVA) in the continuous phase, a tube can be expelled from the rear of the DEs by tip streaming from which smaller daughter droplets are ejected. We imaged the dynamic process of DE formation using 
bright field and fluorescence microscopy inside and at the exit of the channel. Our results show that stable DEs can be generated using such a method and the daughter droplets are aqueous compartments stabilized by DMPC. To the best of our knowledge, this is the first demonstration on tip streaming using phospholipids as a surfactant, which points out the major role played by a bilayer rather than a single layer acting as limit surface and improves the possible comparison with cell-like compartments.

\section{MATERIALS \& METHODS}

Chemicals: Chloroform, polyvinyl alcohol (PVA, Mw $18 \mathrm{kDa}$ ), $\alpha$-hemolysin and fluorescein were supplied by Sigma-Aldrich. DMPC was supplied by Lipoids, Inc. L- $\alpha$-PhosphatidylethanolamineN-(lissamine rhodamine B sulfonyl) (Ammonium Salt) was supplied by Avanti Polar Lipids. All chemicals were used as received.

Double emulsion generation: The double emulsions were generated using a microfluidic flow focusing device consisting of coaxially aligned glass capillary tubes assembled using commercially available fittings, i.e., T-crosses and ferules. The absence of any glued sealing enables not only a fast assembly procedure, but also provides the opportunity of fast replacement of clogged or damaged capillary tubes. The microfluidic device consists of coaxially aligned glass tubes of various dimensions and geometry. First, polyimide coated 365/75 $\mu \mathrm{m}$ (outer diameter $[\mathrm{OD}] /$ inner diameter [ID]) glass capillary tubes (Polymicro) are inserted into a cylindrical 1/0.5 $\mathrm{mm}(\mathrm{OD} / \mathrm{ID})$ borosilicate tube (Drummond Scientific). The borosilicate tube was narrowed in one end for enhanced flow focusing by exposing the tube orifice to a butane flame while rotating the tube. The inner wall and the orifice of this tube were rendered hydrophobic using $2 \%(\mathrm{v} / \mathrm{v})$ trichloro(1H,1H,2H,2H-perfluorooctyl) silane (Sigma-Aldrich) in toluene, rinsed with toluene and 
dried overnight at $70{ }^{\circ} \mathrm{C}$ to obtain the desired wetting properties. These tubes are in turn inserted into a square borosilicate tube $1.25 \times 1 \mathrm{~mm}$ (outer/inner side length, Vitrocom). A second cylindrical borosilicate tube, likewise flame treated and serving as the DE collector tube, was inserted from the opposite end of the square tube, and the two cylindrical tubes were positioned with the narrowed orifices within close distance, followed by positioning of the two inner most fused capillary tubes close to the orifice of the left cylindrical tube. All glass tubes were assembled using T-crosses (P-727, Upchurch Scientific), ferrules (F-331, Upchurch Scientific), and pieces of 1/3 mm (OD/ID) silicon tubing (Tygon Versilic Silicon Tubing, Sigma-Aldrich), and mounted on a Plexiglas stand. A more thorough description of this microfluidic device has been published elsewhere. ${ }^{33}$

Bright field and fluorescence imaging: A Zeiss Axiovert 200 microscope mounted with a NeoFluar objective (2.5x magnification and field depth of $62.4 \mu \mathrm{m})$ and a Dasla CCD camera, was employed for monitoring the DEs. For monitoring the fluorescent probes, a FluoArc laser was connected to the microscope, and the appropriate wavelengths selected with optical band pass filters (532 nm for rhodamine B and $445 \mathrm{~nm}$ for fluorescein). Bright field images were recorded with 300 frames/s, with an exposure time of $3000 \mu s$. Dynamic fluorescence images were recorded with 100 frames/s, with an exposure time of $9000 \mu$ s. Static fluorescence images were recorded with 10 frames/s, with an exposure time of $100 \mathrm{~ms}$. Freely available software ImageJ was used for image processing.

Interfacial tension measurements: An interfacial tensiometer (DSA100, Krüss GMBH), mounted with a 0.516 or a $1.837 \mathrm{~mm}$ needle, was used for measuring the interfacial tension between oil and aqueous phases, employing the pendant drop method with the oil phase suspended in the aqueous phase. 
Viscosity measurements: Viscosities of the PVA solutions were measured using an Anthon Paar Automated Micro Viscometer, employing the rolling ball method, using a $1.6 \mathrm{~mm}$ for the $2 \%$ PVA solution, and a $1.8 \mathrm{~mm}$ capillary tube for $5-10 \%$ solutions. Rolling time was $10 \mathrm{~s}$. The measured viscosity values were found to be $1.63,3.62,6.97$ and $12.94 \mathrm{mPas}$ for 2, 5, 7.5 and 10\% PVA, respectively, see Figure S4.

a-hemolysin studies: $\alpha$-hemolysin (Sigma Aldrich) was incorporated in the DMPC membranes by collecting the double emulsions on a glass slide mounted with a silicon reservoir filled with an aqueous $2 \%$ PVA solution and $\alpha$-hemolysin at a concentration of $50 \mu \mathrm{g} / \mathrm{mL}$.

\section{RESULTS \& DISCUSSION}

The w/o/w double emulsions studied in this work are generated using a co-flow microfluidic channel, ${ }^{33}$ as shown in Figures S1 and S2. The oil phase is constituted of pure chloroform, supplemented with various concentrations of 1,2-dimyristoyl-sn-glycero-3-phosphocholine (DMPC) as the phospholipid (See Materials \& Methods and SI for more details). Poly-vinylalcohol (PVA) is added to the aqueous phases in order to modify the values of viscosity. The interfacial tensions and viscosities of the different formulations are plotted in Figures S3 and S4, respectively. The measurements indicate that the interfacial tension at same DMPC concentration is independent on the concentration of PVA, for $[\mathrm{PVA}] \geq 1 \% \mathrm{w} / \mathrm{w}$ reaching asymptotic values of $0.27,0.18$ and $0.13 \mathrm{mN} . \mathrm{m}^{-1}$ for DMPC concentrations of $0.1,0.5$ and $1.0 \%$, respectively. In contrast, the PVA can be used to efficiently modify the viscosity of the aqueous phase. The shape of each droplet is determined by a balance between the interfacial and viscous forces, as described by the Capillary number $\mathrm{Ca}=\mu \mathrm{U} / \gamma$, where the parameters are characteristic values of the interfacial tension $(\gamma)$, the flow velocity $(\mathrm{U})$, and the viscosity $(\mu)$. The DE droplets are monitored using bright field and fluorescence microscopy at various positions downstream of the point of confluence in the microfluidic device (Figure S1), where they are formed (Figure S2 \& video 1). 
Fluorescein is added to the inner aqueous phase, in order to visualize the core of the droplet, while rhodamine B labelled phospholipids are used as dopants of DMPC in order to image the oil shell. Accordingly, the core drops appear as green, while the red color indicates the phospholipids within the oil shell.

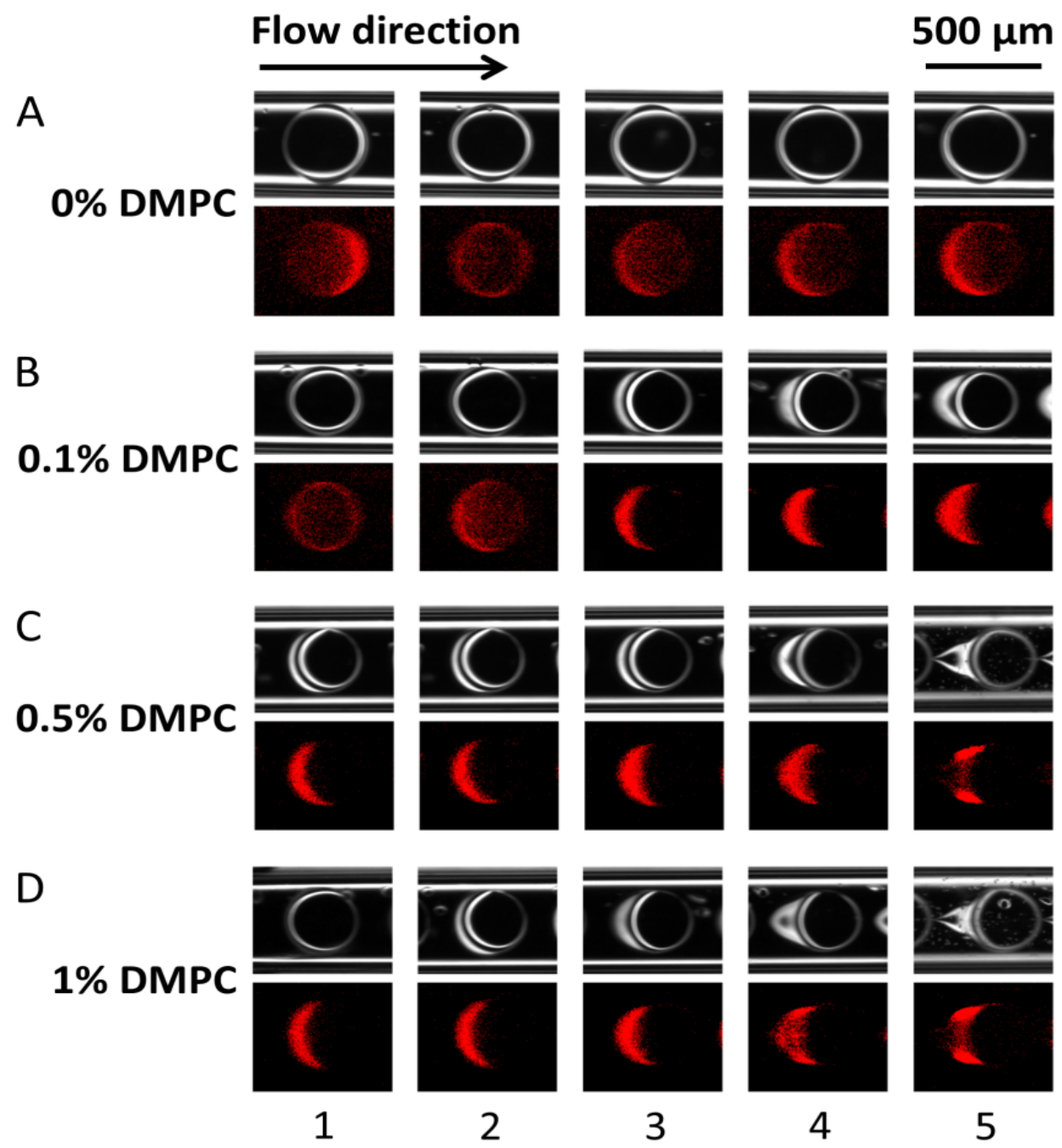

Figure 1. Bright field (top) and fluorescence (bottom) images recorded along the exit tube of the microfluidic device, at distances of (1) $0.2 \mathrm{~mm}$, (2) $5.5 \mathrm{~mm}$, (3) $11 \mathrm{~mm}$, (4) $16.5 \mathrm{~mm}$ and (5) 22 $\mathrm{mm}$ from the point of droplet formation when $2 \% \mathrm{w} / \mathrm{w}$ PVA is used as an internal and external aqueous phase. The concentrations of DMPC in the oil phase are $0,0.1,0.5$ and $1 \%(\mathrm{w} / \mathrm{w})$ in $(\mathrm{A})$, (B), (C) and (D), respectively. Inner diameter of the tube is $500 \mu \mathrm{m}$. Flow rates applied: $\mathrm{Q}_{1}$ $\left(\mathrm{H}_{2} \mathrm{O} / 2 \% \mathrm{w} / \mathrm{w}\right.$ PVA and $0.02 \% \mathrm{w} / \mathrm{w}$ fluorescein, see Figure S5) $=4.17 \times 10^{-4} \mathrm{~cm}^{3} \mathrm{~s}^{-1}, \mathrm{Q}_{2}$ (chloroform/various concentrations of DMPC and $0.02 \% \mathrm{w} / \mathrm{w}$ rhodamine $\mathrm{B}$ labelled phospholipids $)=1.67 \times 10^{-4} \mathrm{~cm}^{3} \mathrm{~s}^{-1}$ and $\mathrm{Q}_{3}\left(\mathrm{H}_{2} \mathrm{O} / 2 \% \mathrm{w} / \mathrm{w} \mathrm{PVA}\right)=4.17 \times 10^{-4} \mathrm{~cm}^{3} \mathrm{~s}^{-1}$. The 
Capillary numbers are $\mathrm{Ca}=2 \times 10^{-3}$, for the case for no DMPC, and $\mathrm{Ca}=6 \times 10^{-2}$ for all other cases. Flow direction is from left to right

The presence and the concentration of DMPC has a major influence on the shape of the DE droplets as they flow through the capillary tube, as shown in Figure 1. Indeed, while the droplets flowing in the absence of DMPC showed slight deformation retaining relatively their concentric spherical shapes (top line of Figure 1A and Figure S5), the addition of even small quantities of phospholipids leads to a stronger deformation of the oil shell that increases progressively as the droplet travels in the capillary tube downstream its point of formation (Figures 1B-D). In the absence of DMPC, the droplets of the double emulsion modify their shape to equilibrate the viscous and capillary effects as they flow. This is also the case in the flow of simple droplets or bubbles. ${ }^{34}$ However, the transition from these small deformations to the singular shape of the tip streaming requires the presence of the phospholipids. The extent of deformation depends on the concentration of DMPC, despite the nominal capillary number remaining constant. Instead, the accumulation of the DMPC at the rear of the droplets, as shown by the red color on the images, indicates that the interface is not at equilibrium, and that this effect depends on the concentration of DMPC. Indeed, the deformation and tip formation become more pronounced as the concentration of DMPC is increased, as shown in Figure 1D, where the outer drop of the DE not only deforms more rapidly, but eventually forms a thin tube at the rear of the DE. This accumulation of DMPC molecules at the rear of the droplet is consistent with the classical models of recirculating flows within rectilinear motion, where the interface motion accumulates partially soluble molecules at the rear end. ${ }^{34}$ Nonetheless, the ability to fluorescently label the phospholipids provides the first direct evidence that this phenomenon is preserved in the case of double 
emulsions. Under all these conditions, the aqueous core of the double emulsion remains concentric, as shown in Figure S5.
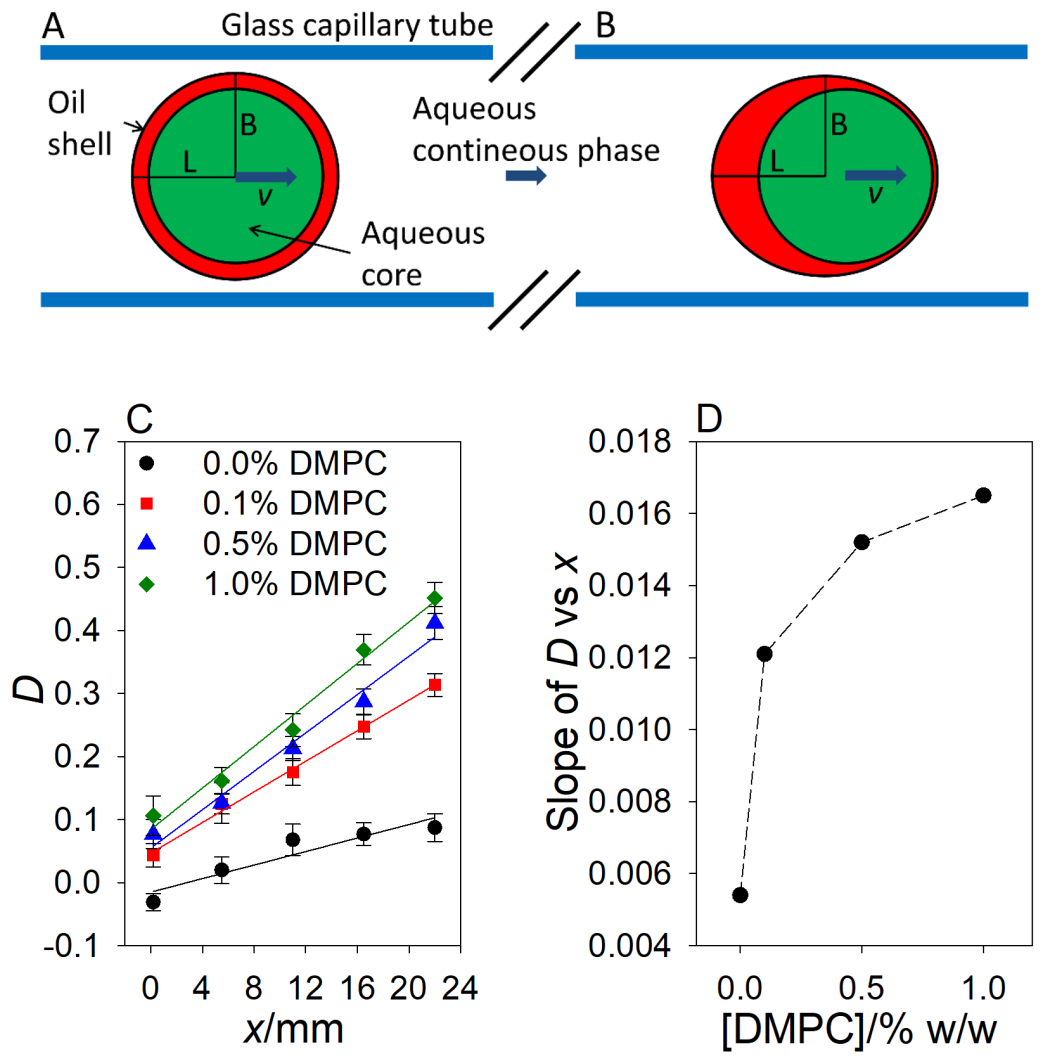

Figure 2. Sketch illustrating the deformation of the middle drop, and the transport of surfactant herein. (A) B and $\mathrm{L}$ are the axial and transverse dimensions, respectively, of the double emulsion moving in the capillary tube with velocity $v$. (B) Intermediate drop deformation, occurring further down the capillary tube, illustrating the rearrangement of the oil phase, and accumulation of surfactant at the rear of the double emulsion. (C) Plot of the deformation parameter D vs. the distance, $x$, travelled by the double emulsion. (D) Extent of deformation, expressed as the slope of the lines in (A), vs. the DMPC concentration. Dashed line in (B) is a guide to the eye. The reported values are the average of three measurements.

To quantify the extent of the deformation (before any observed tip streaming), approximated as the transformation of a sphere to an ellipsoid, we adapt the dimensionless index $\mathrm{D},{ }^{35}$ defined as $D$ 
$=(L-B) /(L+B)$, where $\mathrm{B}$ and $\mathrm{L}$ are the axial and transverse dimensions of the middle droplet (i.e., the oil shell), respectively, as outlined in Figures $2 \mathrm{~A} \& 2 \mathrm{~B}$. Values for B and L are determined from the bright field images of Figure 1. A linear relationship between D and the distance (x) travelled by the DEs is observed for all the applied concentrations of phospholipid, see Figure 2C. This means that a gradual deformation occurs as the droplet advances. The rate of increase of this deformation is plotted in Figure 2D and shows that the deformation rate also increases for higher concentrations of DMPC.

The results of Figures 1 and 2 show that the higher concentrations of DMPC not only lead to larger deformation of the interface, they also increase the rate at which the deformation happens. Taken all together these observations are an indication of the depletion of DMPC molecules in the oil phase at the lower DMPC concentrations, while the higher DMPC concentrations maintain a lower global surface tension even as the molecules are swept to the rear of the droplet. This picture, in turn, is suggestive of a saturation threshold for DMPC in the oil phase.

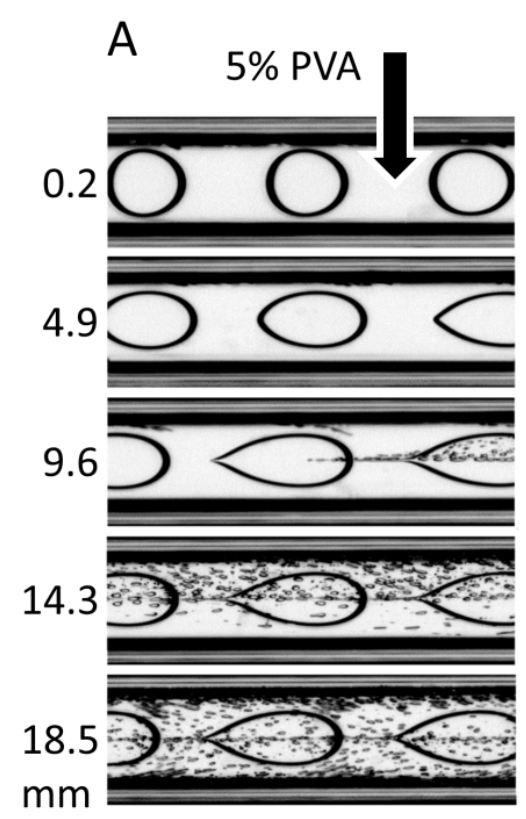

Flow direction

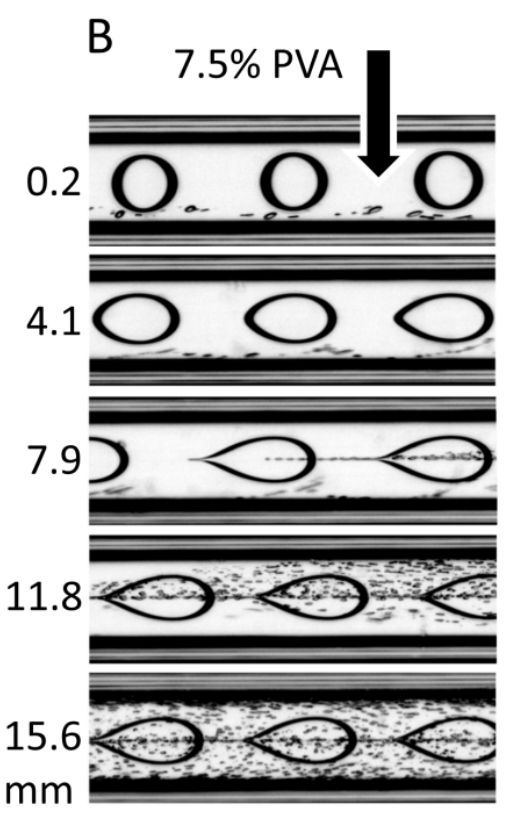

$\mathrm{mm}$

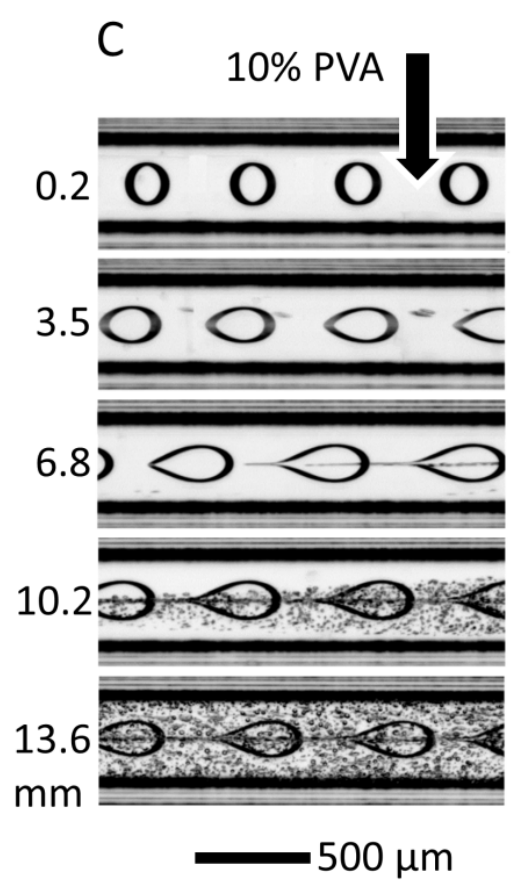


Figure 3. Bright field images showing the gradual deformation of the double emulsion, as an effect of the increasing viscosity of the continuous flowing phase. Each column represents the situation obtained with the PVA concentration indicated. Each frame of the columns is recorded at different locations in the capillary tube. The numbers to the left of each column shows the event location in the tube as the distance from the point of formation of the double emulsions. Flow direction is from left to right. Flow rates applied: $\mathrm{Q}_{1}\left(\mathrm{H}_{2} \mathrm{O} / 2 \% \mathrm{w} / \mathrm{w} \mathrm{PVA}\right)=4.17 \times 10^{-4} \mathrm{~cm}^{3} \mathrm{~s}^{-1}, \mathrm{Q}_{2}$ $($ chloroform $/ 1 \% \mathrm{w} / \mathrm{w}$ DMPC $)=1.67 \times 10^{-4} \mathrm{~cm}^{3} \mathrm{~s}^{-1}$ and $\mathrm{Q}_{3}\left(\mathrm{H}_{2} \mathrm{O} /\right.$ various concentration of PVA $)=$ $4.17 \times 10^{-4} \mathrm{~cm}^{3} \mathrm{~s}^{-1}$. Capillary numbers are $\mathrm{Ca}=0.14$ for the case $5 \% \mathrm{w} / \mathrm{w} \mathrm{PVA}, \mathrm{Ca}=0.3$ for the case $7.5 \% \mathrm{w} / \mathrm{w}$ PVA and $\mathrm{Ca}=0.5$ for the case $10 \% \mathrm{w} / \mathrm{w}$ PVA.

In order to evaluate the relative effect of viscosity change while maintaining the time/space relation as the droplets flow in the channel, the flow rates were kept fixed while the concentration of PVA present in the outer aqueous phase was increased to $5,7.5$, or $10 \%$. Increasing the viscosity leads to a higher dripping frequency and consequently a smaller droplet size. ${ }^{33}$ The resulting deformations and tip streaming are shown in Figure 3, where the inner water droplets display much larger deformation than the case of Figure 1. Indeed, the characteristic capillary numbers for these experiments are in the range $1.4-5.0 \times 10^{-1}$, which are large values compared with other typical microfluidic flows, particularly due to the very low value of the interfacial tension at the interface between chloroform/DMPC and water. These high values of $\mathrm{Ca}$ partially account for the large deformations of the inner water phase. Moreover, the velocity profiles in the different aqueous phases in Figure 3 are expected to be significantly different from the profile in Figure 1 because of the modified viscosity contrast between the outer and inner phases. Indeed, numerical work (for simple droplet flows) has already shown that the flow profile depends on the viscosity ratio between the inner and outer phases. ${ }^{36}$ As such, increasing the viscosity of the outer phase would naturally lead to a different stress field on the flowing droplets than having a viscosity ratio of unity. Here, both the oil shell and the core undergo deformation, which gradually increases until tubes are extruded at the rear end, eventually break up, as displayed in Figure 3 (see Videos 2-4). 
The extent of deformation by varying the concentration of PVA was quantified by plotting D (as defined previously) $v s$. the position (x) in the channel (Figure S6. a). The results show a linear and a gradual deformation of the DEs for a given concentration of PVA upon travelling in the channel with the highest deformation obtained for 10\% PVA as deduced from the slopes of the deformation curves (Figure S6. b). Upon increasing the concentration of PVA which translates in an increase of the capillary number, the deformation increases as shown in Figure S.6.C. These results are in good agreement with previous reports in the literature where high capillary numbers were shown to induce deformation and break-up of simple or double emulsion droplets. ${ }^{25-30}$ The various experimental conditions and regimes observed in this study are summarized in the phase diagrams plotted in Figure S7. They show that a transition from deformation to tip streaming and break-up can occur when $\mathrm{Ca}$ reaches a value below $\sim 0.6$. The deformation of double emulsions subjected to a shear flow was studied experimentally and using numerical simulations by Chen et al. ${ }^{26-27}$ who evidenced four types of breakup modes depending on the capillary number. A breakup via a mechanism based on capillary instability was reported for large capillary numbers as observed in this work. To identify what is being streamed behind the mother droplet, bright field and fluorescence images are acquired at different positions in the capillary tube for the case of $5 \% \mathrm{w} / \mathrm{w}$ PVA in the continuous phase. The higher intensity of red color at the rear of the drops shows that the phospholipids are accumulating at this position (Figure 4A2). At a distance downstream of the position where the tip-streaming has initiated (Figure 4B2) the daughter droplets appear green in the fluorescent image, which indicates that they remain compartmentalized within the outer aqueous phase. The absence of the red signal stemming from the rhodamine B labelled phospholipids in this case is ascribed to the low concentration in the thin oil shell encapsulating 
the daughter droplets, insufficient to be captured with the low exposure time used for the dynamic recordings.
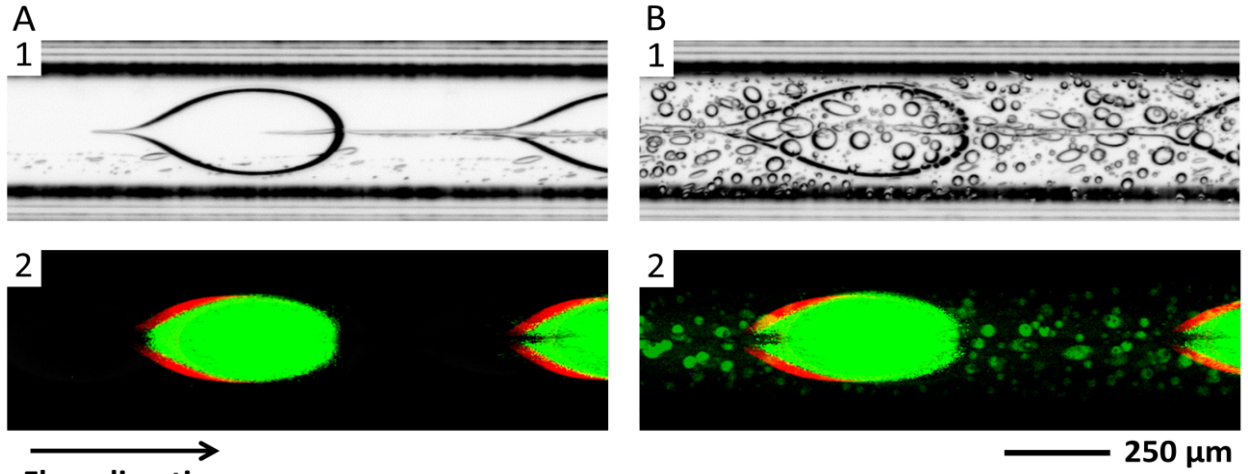

$\overrightarrow{\text { Flow direction }}$

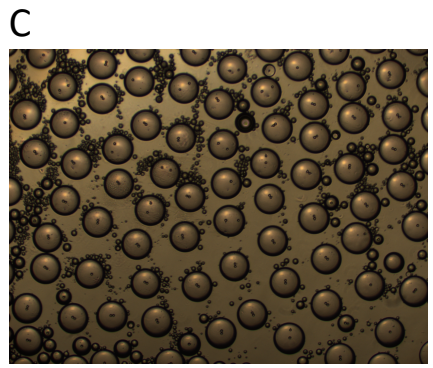

$\mathrm{F}$

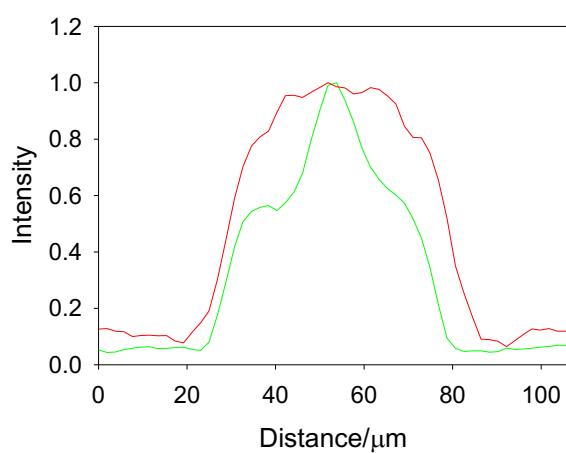

$E$

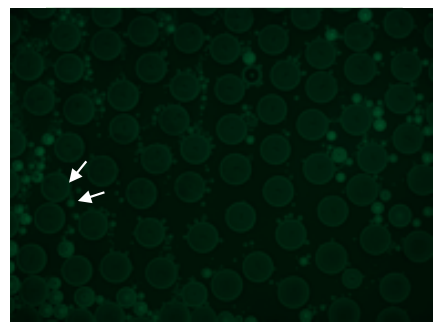

G

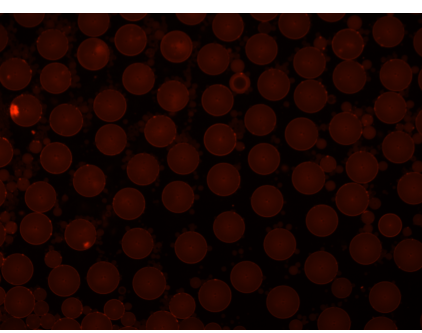

$200 \mu \mathrm{m}=$

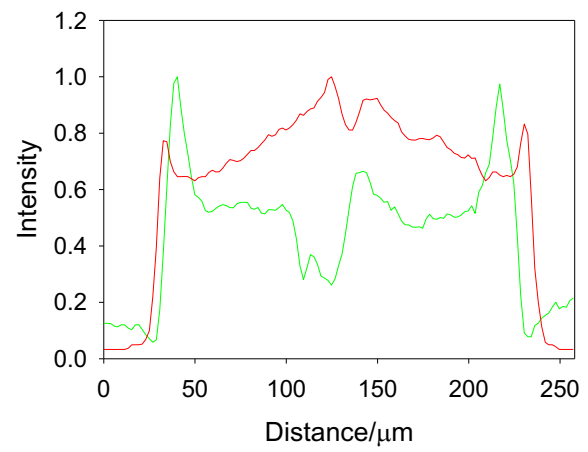

Figure 4. Bright field (1) and fluorescence (2) images of (A) prior to tip-streaming and (B) at a position where tip-streaming has been initiated. The contrast of the green color channel was enhanced to make the daughter droplets appear clearly in image B2. Flow rates applied: $\mathrm{Q}_{1}$ $\left(\mathrm{H}_{2} \mathrm{O} / 2 \% \mathrm{w} / \mathrm{w}\right.$ PVA and $0.02 \% \mathrm{w} / \mathrm{w}$ fluorescein) $=4.17 \times 10^{-4} \mathrm{~cm}^{3} \mathrm{~s}^{-1}, \mathrm{Q}_{2}$ (chloroform $/ 1 \% \mathrm{w} / \mathrm{w}$ DMPC and $0.02 \% \mathrm{w} / \mathrm{w}$ rhodamine $B$ labelled phospholipids $)=1.67 \times 10^{-4} \mathrm{~cm}^{3} \mathrm{~s}^{-1}$ and $\mathrm{Q}_{3}\left(\mathrm{H}_{2} \mathrm{O} / 5 \%\right.$ $\mathrm{w} / \mathrm{w}$ PVA $)=1.67 \times 10^{-4} \mathrm{~cm}^{3} \mathrm{~s}^{-1} . \mathrm{Ca}=0.10$. See also videos $5 \& 6 .(\mathrm{C}, \mathrm{D}$ and $\mathrm{E})$ shows a bright field image, fluorescein encapsulated in the core, and rhodamine B labelled phospholipids in the oil shell, respectively, of mother and daughter droplets collected on a glass slide. Normalized fluorescence intensity plot profiles for fluorescein (green line) and rhodamine B labelled phospholipids (red line) on daughter droplet (F; white arrow) and mother droplet (G; white arrow). 
At the exit of the microchannel the droplets were collected and analyzed by bright field and by fluorescence microscopy at long exposure times. The bright field image (Figure 4C) shows the presence of monodisperse mother droplets with a size around $250 \mu \mathrm{m}$ and smaller droplets generated from the tip streaming with a size ranging from $5-30 \mu \mathrm{m}$. On the top of the mother droplets, small bubbles (dots) are observed which can be attributed to the evaporation of chloroform from the oil shell. The mother and the daughter droplets both exhibit fluorescence signals from the fluorescein in the aqueous core and the rhodamine B labelled phospholipids in the oil shell as shown by fluorescence microscopy, Figure 4D and E, respectively. These results demonstrate clearly that both types of droplets are compartments delimited from the external aqueous phase by a chloroform shell containing phospholipids, and that the daughter droplets inherit from the encapsulated material as well as the oil/phospholipid material. This is also confirmed by the plot profile of the intensity of fluorescence of fluorescein and rhodamine (Figures 4F \& G) across a mother and daughter droplets (indicated by arrows) measured using Image J.

A $\quad \mathbf{t}=\mathbf{0} \mathrm{min}$

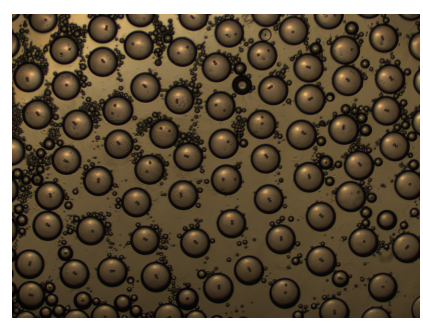

B $\quad \mathbf{t}=15 \mathrm{~min}$

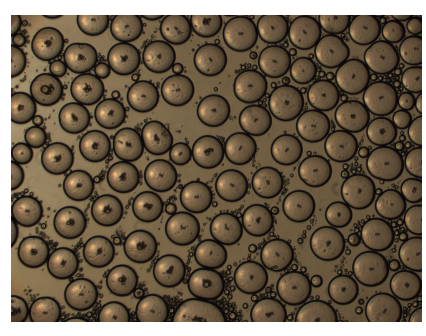

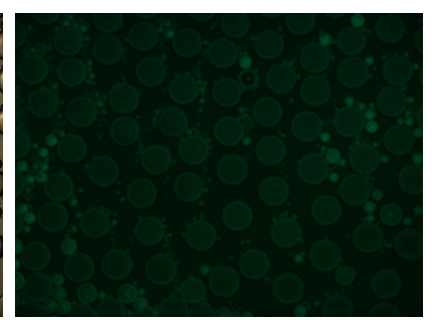

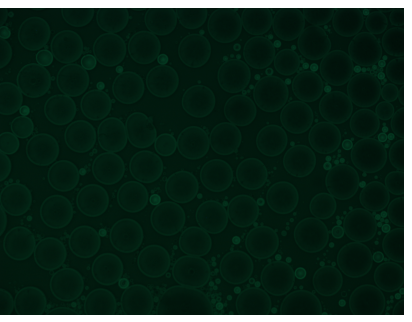

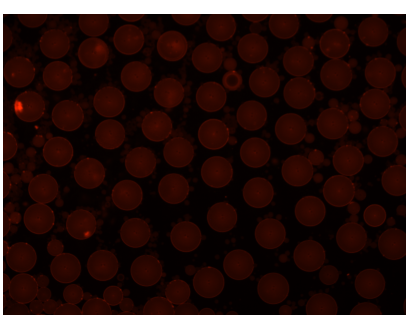

$500 \mu \mathrm{m}$

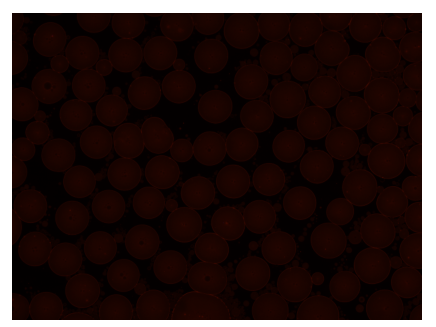

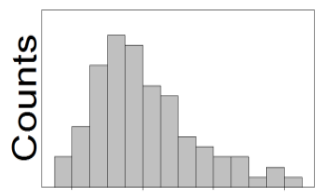

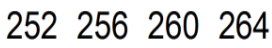

Size/ $\mu \mathrm{m}$

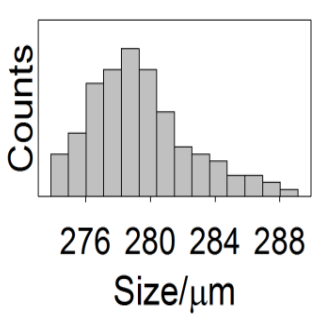


Figure 5. Images showing the effect of membrane incorporated $\alpha$-hemolysin (from left to right: bright field, rhodamine $\mathrm{B}$ fluorescence and fluorescein fluorescence). Images recorded immediately after addition of $\alpha$-hemolysin (A), and after 15 min of incubation with $\alpha$-hemolysin (B) in a $2 \% \mathrm{w} / \mathrm{w}$ PVA matrix. The droplets were generated using the same conditions as described for Figure 4. The histograms display the size increase (swelling) induced by the diffusion of water into the core of the vesicles.

To verify the formation of lipid membranes, we encapsulated fluorescein and $0.2 \mathrm{M}$ sucrose in the inner core of the double emulsions (along with $2 \% \mathrm{w} / \mathrm{w}$ PVA) prior to tip-streaming as previously described. ${ }^{13,37}$ The resulting droplets were collected in a 2\% w/w PVA solution (blank), or in 2\% w/w PVA solution containing $50 \mu \mathrm{g} / \mathrm{mL} \alpha$-hemolysin. The latter spontaneously incorporates into the phospholipid membrane, forming narrow pores. The as formed pores allow fluorescein to exit the droplet core due to the concentration gradient, and water to enter from the exterior matrix due to an osmotic pressure gradient caused by the encapsulated sucrose which will cause the droplets to swell. After 15 min of incubation in the absence of $\alpha$-hemolysin, an increase in the size of $\sim 1 \%$ is observed, lying within the statistical uncertainty, indicating that water does not cross the membrane from the outer to the inner phase. Also, no increase in the fluorescence intensity is observed in the external phase indicating that no passage of fluorescein from the inner phase to the external phase has occurred (most right image, Figure 5A). However, an incubation time of 15 min in the $\alpha$-hemolysin solution resulted in an increase in fluorescence intensity outside the droplets (most right image, Figure $5 \mathrm{~B}$ ) and an increase of $\sim 8 \%$ in size for mother and daughter droplets, with an average size of the mother droplets passing from $256 \pm 14.5 \mu \mathrm{m}$ initially $(57 \pm 18 \mu \mathrm{m}$ for daughter droplets $)$ to a final size of $279 \pm 16 \mu \mathrm{m}(62 \pm 20 \mu \mathrm{m}$ for daughter droplets $)$ as illustrated by the histograms in Figure 5. These results provide evidence that the all obtained compartments are phospholipid vesicles. 


\section{CONCLUSIONS}

In summary, the dynamic behavior of $\mathrm{w} / \mathrm{o} / \mathrm{w}$ double emulsions generated in a microfluidic device, using the phospholipid 1,2-dimyristoyl-sn-glycero-3-phosphocholine (DMPC) as a phospholipid and chloroform as the oil phase is reported in this manuscript. We show that using such formulation, the dynamic behavior of the double emulsions under flow gives rise to strong deformation and tip-streaming. Although such a behavior is well known for droplets formulated using surfactants, it has not been reported neither with double emulsions nor with phospholipids. When the double emulsions were generated using the same composition and viscosity in the core and the outer continuous flowing phase, the concentration of DMPC had a strong impact on the nature and degree of deformation of the middle phase: the shape of the core remained practically unperturbed, while increasing the DMPC concentration resulted in the double emulsions adapting a pear-like shape. Indeed, the higher the DMPC concentration destabilized faster and displayed strong tip-streaming at the rear of the flowing droplets. In this situation it is possible that stacking of DMPC bilayers was less efficient at higher lipid concentration, due to increasing disorder in multiple layers, with resulting unfavorable compartmentalization. The tubes that thus emerged were gradually extruded at the rear of the double emulsions. They eventually pinch off at the tube extremity, in typical form of tip-streaming, as smaller (daughter) compartments were released upon evaporation of chloroform in the form of phospholipid vesicles. The presence of daughter droplets due to trip streaming may be considered as the main disadvantage of this method for the purpose of encapsulation. However, in other cases it can be seen as an advantage to generate very small phospholipid vesicles, which may eventually be expelled from the mother structures, as it happens for instance in cell budding. Centrifugation can be used to separate the vesicles by size or other methods depending on the encapsulated material such as magnetophoresis for magnetic vesicles. Also, our system may serve as a biomimetic model to mimic the generation of extravesicles from cells which can be also used for studying interesting steps in the cell cycle. ${ }^{38-39}$ In the future we would like investigate more in detail the mechanical and fluid-dynamical aspects of this phenomenon, by applying other stimulus which can affect the surface tension, including light, by using photoactivatable molecules (phospholipids, surfactants) ${ }^{40}$ and magnetic fields ${ }^{41}$ (by encapsulation of magnetic particles) on inducing and manipulating the tip streaming under a hydrodynamic flow. 


\section{ASSOCIATED CONTENT}

Supporting Information. Microfluidic generation of the double emulsions, viscosity measurements, surface tension measurements, fluorescence and bright field images, videos on DE generation, PVA viscosity effect, phase diagrams.

\section{AUTHOR INFORMATION}

\section{Corresponding Author}

Ali Abou-Hassan

ali.abou_hassan@sorbonne-universite.fr

Present Addresses

† New affiliation: Université de Paris, Laboratoire d'Electrochimie Moléculaire, UMR CNRS P7 7591, 75205 Paris Cedex 13 - France

\section{Author Contributions}

The manuscript was written through contributions of all authors. All authors have given approval to the final version of the manuscript.

\section{Funding Sources}

Any funds used to support the research of the manuscript should be placed here (per journal style).

\section{ACKNOWLEDGMENT}


K. T and A.A.-H acknowledge F. Brochard Wyart and Patrica Basserau, Institut Curie and

Sorbonne university for fruitful discussions, and the doctoral school ED 398 Chimie Physique et

Chimie Analytique de Paris Centre for funding.

\section{REFERENCES}

1. Shang, L.; Cheng, Y.; Zhao, Y., Emerging Droplet Microfluidics. Chem. Rev. 2017, 117, 7964-8040.

2. $\quad$ Suea-Ngam, A.; Howes, P. D.; Srisa-Art, M.; deMello, A. J., Droplet Microfluidics: From Proof-of-Concept to Real-World Utility? Chem. Commun. 2019, 55, 9895-9903.

3. $\quad$ Kane, R. S.; Stroock, A. D.; Li Jeon, N.; Ingber, D. E.; Whitesides, G. M., Chapter 18 Soft Lithography and Microfluidics. In Optical Biosensors, Ligler, F. S.; Taitt, C. A. R., Eds. Elsevier Science: Amsterdam, 2002; pp 571-595.

4. $\quad$ Utada, A. S.; Lorenceau, E.; Link, D. R.; Kaplan, P. D.; Stone, H. A.; Weitz, D. A., Monodisperse Double Emulsions Generated from a Microcapillary Device. Science 2005, 308, 537-541.

5. Abou-Hassan, A.; Dufrêche, J.-F.; Sandre, O.; Mériguet, G.; Bernard, O.; Cabuil, V., Fluorescence Confocal Laser Scanning Microscopy for Ph Mapping in a Coaxial Flow Microreactor: Application in the Synthesis of Superparamagnetic Nanoparticles. J. Phys. Chem. C. 2009, 113, 18097-18105.

6. Mashaghi, S.; Abbaspourrad, A.; Weitz, D. A.; van Oijen, A. M., Droplet Microfluidics: A Tool for Biology, Chemistry and Nanotechnology. TrAC, Trends Anal. Chem. 2016, 82, 118125 .

7. Ferraro, D.; Lin, Y.; Teste, B.; Talbot, D.; Malaquin, L.; Descroix, S.; Abou-Hassan, A., Continuous Chemical Operations and Modifications on Magnetic $\Gamma$-Fe2o3 Nanoparticles Confined in Nanoliter Droplets for the Assembly of Fluorescent and Magnetic Sio2@ $\Gamma-F e 2 o 3$. Chem. Commun. 2015, 51, 16904-16907.

8. Baroud, C. N.; Gallaire, F.; Dangla, R., Dynamics of Microfluidic Droplets. Lab Chip 2010, 10, 2032-2045.

9. Casadevall i Solvas, X.; deMello, A., Droplet Microfluidics: Recent Developments and Future Applications. Chem. Commun. 2011, 47, 1936-1942.

10. Ding, S.; Serra, C. A.; Vandamme, T. F.; Yu, W.; Anton, N., Double Emulsions Prepared by Two-Step Emulsification: History, State-of-the-Art and Perspective. J Control Release 2019, 295, 31-49.

11. Bhattacharya, A.; Devaraj, N. K., Tailoring the Shape and Size of Artificial Cells. ACS Nano 2019, 13, 7396-7401.

12. Okushima, S.; Nisisako, T.; Torii, T.; Higuchi, T., Controlled Production of Monodisperse Double Emulsions by Two-Step Droplet Breakup in Microfluidic Devices. Langmuir 2004, 20, 9905-9908.

13. Arriaga, L. R.; Datta, S. S.; Kim, S.-H.; Amstad, E.; Kodger, T. E.; Monroy, F.; Weitz, D. A., Ultrathin Shell Double Emulsion Templated Giant Unilamellar Lipid Vesicles with Controlled Microdomain Formation. Small 2014, 10, 950-956.

14. Ho, K. K. Y.; Lee, L. M.; Liu, A. P., Mechanically Activated Artificial Cell by Using Microfluidics. Scientific Reports 2016, 6, 32912 
15. Tomasi, R.; Noel, J.-M.; Zenati, A.; Ristori, S.; Rossi, F.; Cabuil, V.; Kanoufi, F.; AbouHassan, A., Chemical Communication between Liposomes Encapsulating a Chemical Oscillatory Reaction. Chem. Sci. 2014, 5, 1854-1859.

16. Habault, D.; Dery, A.; Leng, J.; Lecommandoux, S.; Meins, J. L.; Sandre, O., Droplet Microfluidics to Prepare Magnetic Polymer Vesicles and to Confine the Heat in Magnetic Hyperthermia. IEEE Transactions on Magnetics 2013, 49, 182-190.

17. Seth, A.; Béalle, G.; Santanach-Carreras, E.; Abou-Hassan, A.; Ménager, C., Design of Vesicles Using Capillary Microfluidic Devices: From Magnetic to Multifunctional Vesicles. Adv. Mater. 2012, 24, 3544-3548.

18. Wang, W.; Zhang, M.-J.; Xie, R.; Ju, X.-J.; Yang, C.; Mou, C.-L.; Weitz, D. A.; Chu, L.Y., Hole-Shell Microparticles from Controllably Evolved Double Emulsions. Angew. Chem. 2013, 125, 8242-8245.

19. Zhang, Y.; Ho, Y.-P.; Chiu, Y.-L.; Chan, H. F.; Chlebina, B.; Schuhmann, T.; You, L.; Leong, K. W., A Programmable Microenvironment for Cellular Studies Via MicrofluidicsGenerated Double Emulsions. Biomaterials 2013, 34, 4564-4572.

20. Torbensen, K.; Rossi, F.; Pantani, O. L.; Ristori, S.; Abou-Hassan, A., Interaction of the Belousov-Zhabotinsky Reaction with Phospholipid Engineered Membranes. The Journal of Physical Chemistry B 2015, 119, 10224-10230.

21. Rossi, F.; Zenati, A.; Ristori, S.; NoÎl, J.-M.; Cabuil, V.; Kanoufi, F.; Abou-Hassan, A., Activatory Coupling among Oscillating Droplets Produced in Microfluidic Based Devices. Int. J. Unconv. Comput. 2015, 11, 23-36.

22. Budroni, M. A.; Torbensen, K.; Pantani, O. L.; Ristori, S.; Rossi, F.; Abou-Hassan, A., Microfluidic Compartmentalization of Diffusively Coupled Oscillators in Multisomes Induces a Novel Synchronization Scenario. Chem. Commun. 2020, 56, 11771-11774.

23. Eggleton, C. D.; Tsai, T.-M.; Stebe, K. J., Tip Streaming from a Drop in the Presence of Surfactants. Phys. Rev. Lett. 2011, 87, 048302-1 - 048302-4.

24. Anna, S. L.; Bontoux, N.; Stone, H. A., Formation of Dispersions Using "Flow Focusing" in Microchannels. Appl. Phys. Lett. 2003, 82, 364-366.

25. Zhao, C.-X.; Middelberg, A. P. J., Two-Phase Microfluidic Flows. Chem. Eng. Sci. 2011, 66, 1394-1411.

26. Hua, H.; Shin, J.; Kim, J., Dynamics of a Compound Droplet in Shear Flow. Int. J. Heat Fluid Flow 2014, 50, 63-71.

27. Chen, Y.; Liu, X.; Shi, M., Hydrodynamics of Double Emulsion Droplet in Shear Flow. Appl. Phys. Lett. 2013, 102, 051609.

28. Chen, Y.; Liu, X.; Zhao, Y., Deformation Dynamics of Double Emulsion Droplet under Shear. Appl. Phys. Lett. 2015, 106, 141601.

29. Ma, S.; Huck, W. T. S.; Balabani, S., Deformation of Double Emulsions under Conditions of Flow Cytometry Hydrodynamic Focusing. Lab Chip 2015, 15, 4291-4301.

30. Zhou, C.; Yue, P.; Feng, J. J., Formation of Simple and Compound Drops in Microfluidic Devices. Physics of Fluids 2006, 18, 092105.

31. Tao, J.; Song, X.; Liu, J.; Wang, J., Microfluidic Rheology of the Multiple-Emulsion Globule Transiting in a Contraction Tube through a Boundary Element Method. Chem. Eng. Sci. 2013, 97, 328-336.

32. Chen, H.; Li, J.; Shum, H. C.; Stone, H. A.; Weitz, D. A., Breakup of Double Emulsions in Constrictions. Soft Matter 2011, 7, 2345-2347. 
33. Torbensen, K.; Abou-Hassan, A., Easy-to-Assemble and Adjustable Coaxial FlowFocusing Microfluidic Device for Generating Controllable Water/Oil/Water Double Emulsions: Toward Templating Giant Liposomes with Different Properties. J. Flow. Chem. 2015, 5, 234240.

34. Ajaev, V. S.; Homsy, G. M., Modeling Shapes and Dynamics of Confined Bubbles. Annual Review of Fluid Mechanics 2006, 38, 277-307.

35. Taylor, G. I., The Formation of Emulsions in Definable Fields of Flow. Proceedings of the Royal Society of London A: Mathematical, Physical and Engineering Sciences 1934, 146, 501-523.

36. Hodges, S. R.; Jensen, O. E.; Rallison, J. M., The Motion of a Viscous Drop through a Cylindrical Tube. J. Fluid Mech. 2004, 501, 279-301.

37. Bao, P.; Paterson, D. A.; Peyman, S. A.; Jones, J. C.; Sandoe, J. A. T.; Gleeson, H. F.; Evans, S. D.; Bushby, R. J., Production of Giant Unilamellar Vesicles and Encapsulation of Lyotropic Nematic Liquid Crystals. Soft Matter 2021, 17, 2234-2241.

38. Mathieu, M.; Martin-Jaular, L.; Lavieu, G.; Théry, C., Specificities of Secretion and Uptake of Exosomes and Other Extracellular Vesicles for Cell-to-Cell Communication. Nature Cell Biology 2019, 21, 9-17.

39. Liu, Q.; Bi, C.; Li, J.; Liu, X.; Peng, R.; Jin, C.; Sun, Y.; Lyu, Y.; Liu, H.; Wang, H.; Luo, C.; Tan, W., Generating Giant Membrane Vesicles from Live Cells with Preserved Cellular Properties. Research 2019, 9, 6523970

40. Diguet, A.; Li, H.; Queyriaux, N.; Chen, Y.; Baigl, D., Photoreversible Fragmentation of a Liquid Interface for Micro-Droplet Generation by Light Actuation. Lab Chip 2011, 11, 26662669.

41. Rigoni, C.; Fresnais, J.; Talbot, D.; Massart, R.; Perzynski, R.; Bacri, J. C.; Abou-Hassan, A., Magnetic Field-Driven Deformation, Attraction, and Coalescence of Nonmagnetic Aqueous Droplets in an Oil-Based Ferrofluid. Langmuir 2020, 36, 5048-5057. 
For Table of Contents Only

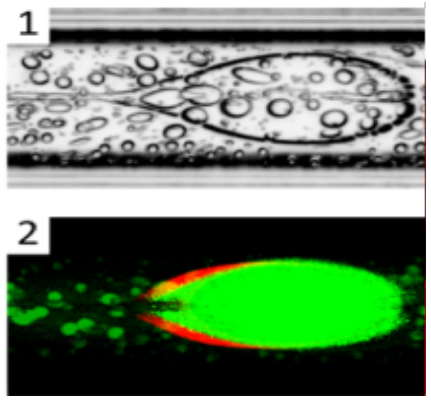

